\title{
DESENVOLVIMENTO DE CÓDIGO COMPUTACIONAL PARA ANÁLISE DINÂMICA DE TURBINAS EÓLICAS E HIDROCINÉTICAS PARA FINS DE ENSINO E PESQUISA
}

\author{
DEVELOPMENT OF COMPUTATIONAL CODE FOR DYNAMIC ANALYSIS OF WIND AND \\ HYDROKINETIC TURBINES FOR TEACHING AND RESEARCH PURPOSES
}

\author{
Denis Melo Rocha ${ }^{1}$, Rodrigo Marques dos Santos ${ }^{2}$, \\ Sérgio de Souza Custódio Filho ${ }^{3}$, Alexandre Luiz Amarante Mesquita ${ }^{4}$ \\ DOI: 10.37702/REE2236-0158.v39p109-122.2020
}

\begin{abstract}
RESUMO
Este artigo descreve o desenvolvimento de um código computacional para análise dinâmica de turbinas eólicas e hidrocinéticas de eixo horizontal usando o software Matlab. A modelagem inclui os aspectos aerodinâmicos (ou hidrodinâmicos) do rotor da turbina acoplado ao comportamento dinâmico do seu trem de potência, que inclui os efeitos da transmissão mecânica e do gerador elétrico. Os momentos de inércia de massa de todos os componentes da turbina são considerados, assim como o torque do rotor, torque dissipativo por atrito e o torque do gerador. A interface com o usuário possui uma faixa de opções na qual modelos matemáticos existentes de torques e momentos de inércia podem ser escolhidos assim como novos modelos podem ser inseridos e avaliados. Para verificar a precisão do programa, um estudo de caso da literatura foi usado e os resultados para rotação e torque da turbina mostraram excelente concordância com os resultados originais. A versatilidade do código computacional o torna bem adequado para fins de ensino e pesquisa, pois o usuário pode facilmente verificar a influência de parâmetros dos modelos matemáticos de torque e de momento de inércia no comportamento dinâmico da turbina, além de poder testar novos modelos.
\end{abstract}

Palavras-chave: turbina eólica; turbina hidrocinética; modelagem computacional.

\begin{abstract}
This paper describes a computational code for dynamic analysis of horizontal axis wind and hydrokinetic turbines using Matlab software. The modeling includes the aerodynamic (or hydrodynamic) aspects of the turbine rotor coupled to its powertrain dynamical behavior, which includes the mechanical transmission and electric generator effects. The mass moments of inertia of all components of the turbine are considered, as well as, the rotor torque, the friction dissipative torque and generator torque. The user interface has a range of options where mathematical models of torques and moments of inertia can be chosen as well as new models can be inserted and evaluated. In order to verify the accuracy of the code, a case study from the literature was used and the results for turbine rotation and torque showed excellent agreement with the original results. The developed system becomes very useful for research and teaching purposes because the user can easily verify the influence of parameters of torque and moment of inertia models on the dynamic behavior of the turbine, and even test new models.
\end{abstract}

Keywords: wind turbine; hydrokinetic turbine; computational modeling.

\footnotetext{
1 Mestrando no Programa de Pós-Graduação em Engenharia Mecânica da Universidade Federal do Pará (UFPA); denisrocha012@gmail.com 2 Graduando em Engenharia Mecânica na Universidade Federal do Pará (UFPA); rodrigomsantos730@ gmail.com

3 Professor Substituto e mestrando no Programa de Pós-Graduação em Engenharia Mecânica da Universidade Federal do Pará (UFPA); engsergiocustodio@gmail.com

${ }_{4}$ Professor Doutor na Universidade Federal do Pará (UFPA); alexmesq@ufpa.br
} 


\section{INTRODUÇÃO}

Turbinas hidrocinéticas são similares às turbinas eólicas, tanto no projeto quanto na operação, ou seja, aproveitam a energia cinética do fluxo do fluido de trabalho e convertem em energia elétrica (LAWS; EPPS, 2016). A geração de energia elétrica por esses dois sistemas cinéticos tem despertado grande interesse em vários países, devido ao fato de que usam fontes renováveis (vento e correnteza de rios e oceanos) com baixos impactos ambientais (KUMAR; SARKAR, 2016). No Brasil, tais tecnologias são bem adequadas devido ao bom potencial eólico existente nas regiões costeiras do país (BLASQUES et al., 2010) e também devido à sua vasta e densa rede hidrográfica.

A maior parte das comunidades não atendidas pelo sistema de distribuição de energia elétrica convencional está localizada na Amazônia (IEMA, 2019). Dessa forma, essa região, com uma extensa área hidrográfica, possui um potencial enorme para instalação de turbinas hidrocinéticas, uma tecnologia ainda em desenvolvimento no Brasil (VAN ELS; JUNIOR, 2015).

Por outro lado, a crescente evolução da energia eólica na composição da matriz energética do Brasil fornece um quadro de demanda de aprofundamento de estudos na tecnologia de turbinas eólicas (aerogeradores). $\mathrm{Na}$ região amazônica, os locais próximos à costa e às elevações (topo dos montes ou montanhas) são, em geral, os que possuem as melhores velocidades médias de vento ao longo de um ano (BLASQUES et al., 2010). Fora da região costeira e de elevações, há a necessidade de adaptação dos aerogeradores para essas baixas condições de vento. Muitos dos equipamentos utilizados nesses sistemas, apesar de fabricados no Brasil, ainda utilizam tecnologias importadas e suas características físicas, em geral, não são apropriadas para essas baixas condições de vento.

Verificando a necessidade de desenvolvimento de tecnologias de turbinas cinéticas e de uma maior capacitação dos engenheiros atuantes na região, pesquisadores da região têm buscado desenvolver várias ações de ensino e pesquisa. No ensino, ações têm sido feitas na Universidade Federal do Pará (UFPA) por meio de ofertas de cursos regulares e extracurriculares, além de atuações voltadas para o ensino fundamental e médio (FIGUEIREDO et al., 2013). Em relação à pesquisa, vários projetos de pesquisa têm sido desenvolvidos, e os pesquisadores locais - em conjunto com pesquisadores de outras instituições (Universidade de Brasília - UnB e Universidade de Calgari/Canadá) - têm desenvolvido modelos matemáticos voltados para a previsão do comportamento dinâmico de turbinas eólicas e hidrocinéticas. Tais modelos têm sido bem aceitos na comunidade científica (VAZ; WOOD, 2018; LOPES et al., 2015; MESQUITA et al., 2014; SILVA et al., 2017) e, portanto, estão sendo difundidos no ensino em disciplinas da graduação e de pósgraduação da Engenharia Mecânica da UFPA. Contudo, verificou-se que para uma melhor assimilação do conteúdo pelos discentes faziase necessária a inclusão de ferramentas computacionais e visuais que facilitassem o processo de ensino e de aprendizagem (MARIANI; MARTIM, 2008).

O Matlab, desenvolvido pela MathWorks5, é um dos softwares mais usados para modelagem e simulação de sistemas dinâmicos (LIMA et al., 2016). O software oferece também uma poderosa ferramenta de programação gráfica, o ambiente GUIDE (Graphical User Interface Development Environment), o qual permite a criação de interessantes e versáteis interfaces gráficas (GUI) pelo usuário.

Dessa forma, este trabalho apresenta o desenvolvimento de um código computacional voltado para o ensino e pesquisa em dinâmica de turbinas eólicas e hidrocinéticas de eixo horizontal. O código foi gerado em Matlab utilizando-se o ambiente GUIDE como ferramenta para criação de interfaces, visando a facilitar a compreensão dos fenômenos envolvidos, tais como o efeito dos parâmetros de torque e momento de inércia no comportamento dinâmico da turbina. $\mathrm{O}$ código computacional implementa o modelo 
matemático apresentado em Mesquita et al. (2014) e Lopes et al. (2015), sendo que cada parâmetro do modelo dinâmico da turbina possui uma opção na interface GUI, de forma que alterações nesses parâmetros possam ser feitas de maneira bem simples. Para cada parâmetro de torque ou de momento de inércia, há a opção de escolha de diferentes modelos matemáticos existentes da literatura. $\mathrm{O}$ programa também permite a inserção de novos modelos matemáticos de torques e de momentos de inércia.

A partir daqui o artigo se organiza da seguinte forma: na seção seguinte é descrito o modelo dinâmico aqui usado para turbinas cinéticas; em seguida, apresenta-se a implementação desse modelo em ambiente Matlab e como as interfaces GUIs são apresentadas; adiante, um estudo de caso é exposto para validação do programa computacional desenvolvido; e, finalmente, as conclusões deste trabalho são apontadas.

\section{MODELAGEM DINÂMICA DE TURBINAS CINÉTICAS DE EIXO HORIZONTAL}

Os principais componentes de uma turbina cinética de eixo horizontal são o rotor da turbina, sistema de transmissão e gerador elétrico, conforme esquema da Figura 1.

Figura 1 - Turbina com seus componentes principais

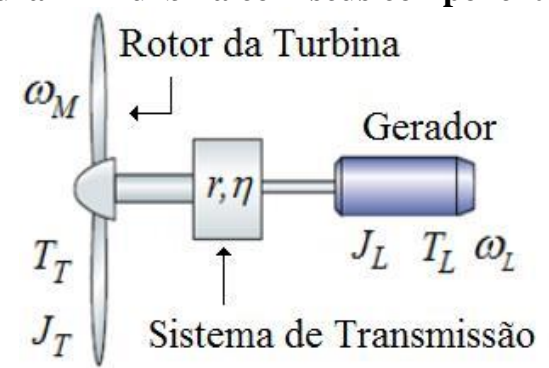

Fonte: acervo dos autores.

Partindo-se da premissa que os eixos são infinitamente rígidos (hipótese válida quando as frequências naturais do sistema são bem maiores do que a sua faixa operacional), o modelo matemático que rege o comportamento dinâmico da turbina é dado pela Equação (1), que é a aplicação da $2^{a}$ Lei de Newton para movimento rotativo:

$$
\begin{aligned}
T_{T}-\left(T_{D, \text { total }}\right. & \left.+T_{L \rightarrow T}\right) \\
& =J_{\text {total }} \frac{d \omega_{M}}{d t}
\end{aligned}
$$

sendo que o torque motriz, $T T$, é o torque do rotor da turbina, subtraído do somatório de todos os torques resistentes $\left(T_{R}\right)$ ao movimento de rotação do sistema: $T_{D \text {,total }}$ (torque dissipativo total nos mancais) e torque equivalente no gerador $T_{L \rightarrow T}$ (torque da carga com referência ao eixo do rotor da turbina). $J_{\text {total }}$ é o momento de inércia de massa de todo o sistema e $\omega m$ é a velocidade angular do rotor da turbina.

Os termos de torque e de momento de inércia de massa da Equação (1) podem ser modelados de diferentes maneiras. Assim, as seções seguintes mostram alguns diferentes modelos encontrados na literatura para cada termo da equação.

\section{Torque do Rotor da Turbina}

A expressão do torque do rotor é obtida a partir do quociente entre a potência mecânica $P_{M}$ do rotor e sua velocidade angular $\omega M$, ou seja:

$$
T_{T}=\frac{P_{M}}{\omega_{M}}
$$

A potência mecânica pode ser escrita como sendo a potência disponível (derivada da energia cinética da massa fluida em relação ao tempo) vezes o coeficiente de potência $C p$ do rotor da turbina, o qual quantifica o quanto da potência disponível é convertida em potência mecânica; dessa forma a Equação (2) torna-se:

$$
T_{T}=\frac{1}{2} \frac{\rho \pi R^{2} V^{3}}{\omega_{M}} C_{p}
$$

sendo $\rho$ a massa específica do fluido no qual a turbina está imersa, $R$ o raio da turbina eólica/hidrocinética e $V$ a velocidade do fluido.

$\mathrm{O}$ valor máximo de $C p$ para uma turbina ideal é o valor limite de Betz, ou seja, $\mathrm{Cp}=59,26 \%$ (HANSEN, 2008). Na prática, alguns efeitos conduzem a uma redução no limite de Betz, efeitos tais como rotação da 
esteira de vórtices a jusante do rotor, número finito de pás e perdas associadas aos efeitos de ponta de pá (HANSEN, 2008).

O $C p$ pode ser determinado pela teoria clássica do BEM (Blade Element Momentum Theory) também referida na literatura como BEMT (DEHOUCK et al., 2018), sendo escrito como:

$$
C_{p}=\frac{8}{\lambda^{2}} \int_{0}^{\lambda} a^{\prime} F(1-a F) x^{3} d x
$$

sendo $a$ o fator de indução axial, $a$ ' o fator de indução tangencial, $F$ a força hidrodinâmica, $\lambda$ a razão de velocidade TSR (Tip-Speed-Ratio) e $x$ a razão de velocidade local LSR (LocalSpeed-Ratio).

\section{Torque Dissipativo}

Os torques dissipativos são todos os torques resistivos ao movimento de rotação da turbina no conjunto rotor-caixa multiplicadoragerador. Para uma turbina hidrocinética considera-se como resistivos os torques de atrito nos mancais e o torque devido à massa aditiva do fluido nas pás da turbina; no entanto, para turbinas eólicas, apenas os torques de atrito nos mancais são considerados, devido à massa específica do ar ser muito menor do que a da água, o que diminui bastante o arrasto no rotor provocado pela viscosidade do fluido. Em relação às perdas associadas na caixa de transmissão, ou seja, nos engrenamentos, elas são levadas em conta por meio do rendimento da transmissão mecânica.

Entre os modelos para o torque dissipativo nos mancais tem-se o modelo de atrito de Coulomb rotativo (BRÄNDLEIN et al., 1999), que é independente da velocidade de rotação e dado por

$$
T_{D, C}=\mu F_{1} \frac{d_{m}}{2}
$$

sendo $\mu$ o coeficiente de atrito, $F_{1}$ a resultante da carga no mancal $F_{1}=\left(F_{\mathrm{a} 2}+F_{r 2}\right)_{1 / 2}$ e $d_{m}$ o diâmetro primitivo ou diâmetro médio (entre o diâmetro interno e o externo) do mancal. Esse torque de atrito pode fornecer uma boa primeira estimativa do torque de atrito em condições bem controladas em regime permanente.
O torque dissipativo dos mancais pode ser dado também em função do tipo do mancal. Por exemplo, no caso de mancais de rolos esféricos, Palmgren (1959) separou o torque dissipativo nos mancais em dois tipos: componente dependente da carga $T_{1}$ e componente independente da carga $T$, o qual é influenciado pelas propriedades viscosas do tipo de lubrificante, pela quantidade de lubrificante aplicado e pela velocidade dos rolamentos. Logo, o torque dissipativo total para mancais esféricos [N.m] é obtido através da seguinte equação:

$$
T_{D, P}=T_{0}+T_{1}
$$

em que

$$
\begin{aligned}
& T_{0}=10^{-10} \times f_{0}\left(n v_{0}\right)^{\frac{2}{3}} d_{m}^{3} \\
& T_{1}=10^{-3} \times f_{1} \times F_{\beta} \times d_{m}
\end{aligned}
$$

sendo $f_{0}$ fator que depende do tipo de mancal e do método de lubrificação utilizado, $n$ a rotação, $v_{0}$ a viscosidade cinemática do lubrificante, $f_{1}$ o fator dependente da geometria do mancal e da carga relativa aplicada no mancal e $F_{\beta}$ o fator dependente da magnitude e direção da carga aplicada.

Também para rolamentos esféricos, a SKF (2015) dispõe em seu catálogo outro tipo de formulação:

$$
T_{D, S K F}=T_{r r}+T_{s l}+T_{\text {seal }}+T_{\text {drag }}
$$

sendo $T_{r r}$ o torque de atrito de rolamento, $T_{s l} \mathrm{o}$ torque de atrito deslizante, $T_{\text {seal }}$ o torque de atrito nos selos e $T_{d r a g}$ o torque de atrito das perdas por arrasto. Cada termo pode ser encontrado com mais detalhes no catálogo da SKF (2015).

Por outro lado, um método simplificado para obter o torque dissipativo é descrito nos trabalhos desenvolvidos por Bao e Ye (2001), através da equação:

$$
T_{D, B}=C_{1}+\frac{C_{2}}{\omega_{M}}+C_{3} \omega_{M}
$$

onde $C_{1}, C_{2}$ e $C_{3}$ são constantes apropriadas devido ao atrito das partes mecânicas impostas ao rotor.

Recentemente, Vaz et al. (2018) utilizaram uma metodologia mais elaborada para o cálculo 
do torque dissipativo. A partir da metodologia desenvolvida pelo fabricante de rolamentos SKF (2015), foi considerado no cálculo o efeito do torque na partida da máquina, $T_{S}-$ Efeito de Stribeck, como demonstrado a seguir:

$$
\begin{gathered}
T_{D, S K F}=T_{S} \cdot \exp \left[-\left(\frac{n}{n_{s t}}\right)^{i}\right] \\
+T_{r r}
\end{gathered}
$$

sendo:

$$
\left.T_{s}\right|_{n=0}=0.5\left(T_{s l}-T_{\text {seal }}\right)
$$

e $n_{s t}$ a velocidade de Stribeck, $i$ o expoente de Stribeck e $T_{r r}$ o torque de atrito de rolamento.

\section{Torque do Gerador}

O torque equivalente do gerador (ou torque equivalente da carga) é o torque da carga com referência ao eixo de maior velocidade e a sua expressão é dada por:

$$
T_{L-T}=\frac{1}{\eta} r T_{L}
$$

sendo que $T_{L}$ é o torque do gerador, $\eta$ é a eficiência da transmissão e $r$ é a razão de velocidade, dada por $r=\omega L / \omega M$.

$\mathrm{O}$ torque do gerador $T_{L}$ pode ser descrito em função dos parâmetros elétricos, como demonstrado em Vásquez, De Oliveira e Junior (2016) para o gerador síncrono e em Reddy e Bhagyamma (2014) para o gerador assíncrono. Entretanto, a equação de torque do gerador pode ser simplificada usando-se dados dos fabricantes. Desse modo, uma relação entre o torque do gerador síncrono e sua velocidade angular pode ser dada por uma equação linear aproximada, como em Bao e Ye (2001), ou seja:

$$
T_{L}=k_{e} \omega_{L}+k_{0}
$$

sendo que $\omega L$ é a velocidade angular do gerador e $k e$ e $k 0$ são coeficientes obtidos por regressão linear ajustada aos dados experimentais da curva do torque do gerador fornecido pelo fabricante do gerador.

\section{Momentos de inércia}

O momento de inércia de massa total do sistema $\left(J_{\text {total }}\right)$ é dado pela seguinte expressão:

$$
J_{\text {total }}=J_{T}+J_{f}+J_{G T}+J_{L \rightarrow T}
$$

sendo $J_{T}$ o momento de inércia de massa do rotor da turbina, $J_{f}$ o momento de inércia da massa aditiva do fluido em torno das pás (para turbinas hidrocinéticas), $J_{G T}$ a inércia da transmissão mecânica (geralmente, uma caixa de engrenagens multiplicadoras) e $J_{L \rightarrow T}$ a inércia equivalente do gerador. Esses momentos de inércias são mais bem descritos abaixo.

O momento de inércia de massa do rotor da turbina corresponde aos momentos de inércias das massas das pás do rotor mais o momento de inércia de massa do cubo do rotor. Existem diferentes modelos matemáticos para a expressão da inércia das pás do rotor. Em Rosales et al. (2013) a inércia das pás do rotor é dada por:

$$
\begin{aligned}
& J_{T} \\
& =N \rho_{b} A R\left[\int(c r)^{2} d r\right. \\
& +\frac{A}{12}\left(\int c^{4} \cos ^{2} \theta_{p} d r\right. \\
& \left.\left.+A^{2} \int c^{4} \operatorname{sen}^{2} \theta_{p} d r\right)\right]
\end{aligned}
$$

sendo $N_{p}$ o número de pás, $\rho b$ a densidade do fluido, $A$ a área da superfície da pá, $R$ o raio total do rotor, $c$ o comprimento da corda em função de $r$ (incremento de distância ao longo do comprimento da pá) e $\theta_{p}$ o ângulo de passo da pá.

Em Mesquita et al. (2014) é desenvolvida uma expressão para calcular o momento de inércia de massa de uma pá, dividindo a pá em finitos volumes ao longo do seu perfil, e em cada volume determina-se o centro de massa e a distância entre o centro de massa e o centro de rotação da pá. O momento de inércia de massa da raiz ou base da pá que pode ser aproximada como um cilindro oco também é considerado. Portanto, a equação que fornece o momento de inércia de massa da pá é dada por:

$$
J_{p a ́}=\sum_{i=1}^{N} m_{i} r_{i}^{2}+m_{\text {base }} r_{\text {base }}^{2}
$$

Outra forma de determinar o momento de inércia de massa das pás pode ser por meio de 
softwares dedicados a desenhos e simulações, tal como o SolidWorks CAD 3D. Nesse software, ao completar o desenho de um sólido, pode-se obter o valor do momento de inércia em relação a um dos três eixos cartesianos. Outra maneira é determinar o momento de inércia da pá experimentalmente, por meio da frequência de oscilação da pá tomada como um pêndulo.

Para turbinas eólicas, a massa aditiva do ar é desprezível. Portanto, a massa aditiva será levada em conta apenas para turbinas hidrocinéticas. Na literatura, há vários modelos para o momento de inércia da massa aditiva, tais como: os modelos de Wilson (1956) e Lewis e Auslaender (1960), que foram desenvolvidos para propulsores navais, mas que podem ser adaptados para rotores de turbinas. Mais recentemente, Maniaci e Li (2012) apresentaram um modelo para a massa aditiva sendo igual à massa de um cilindro (de comprimento igual ao comprimento da pá) cujo diâmetro é igual ao comprimento da corda.

Para o cálculo do momento de inércia de massa da transmissão, pode-se utilizar o princípio de que a energia cinética equivalente de um sistema é igual ao somatório da energia cinética de cada componente da transmissão, a qual é calculada de acordo com o tipo de movimento do corpo (translação, rotação ou movimento plano geral). Como exemplo, abaixo tem-se o momento de inércia de uma transmissão por engrenagens planetárias (Figura 2), bastante usada em turbinas eólicas e hidrocinéticas:

$$
\begin{aligned}
& J_{S T} \\
& =J_{B} \\
& +N_{P}\left[J_{P}\left(\frac{R_{S}+R_{P}}{R_{P}}\right)^{2}\right. \\
& \left.+m_{P}\left(R_{S}+R_{P}\right)^{2}\right] \\
& +\left[\frac{2\left(R_{S}+R_{P}\right)}{R_{S}}\right]^{2} J_{S}
\end{aligned}
$$

sendo $J_{B}$ a inércia do braço das engrenagens planetas, cuja quantidade é dada por $N P$, inércia $J_{P}$, massa $m P$ e raio $R_{P}$. O raio da engrenagem sol é dado por $R S$.
Figura 2 - Representação de uma turbina hidrocinética de eixo horizontal com transmissão por engrenagens planetárias

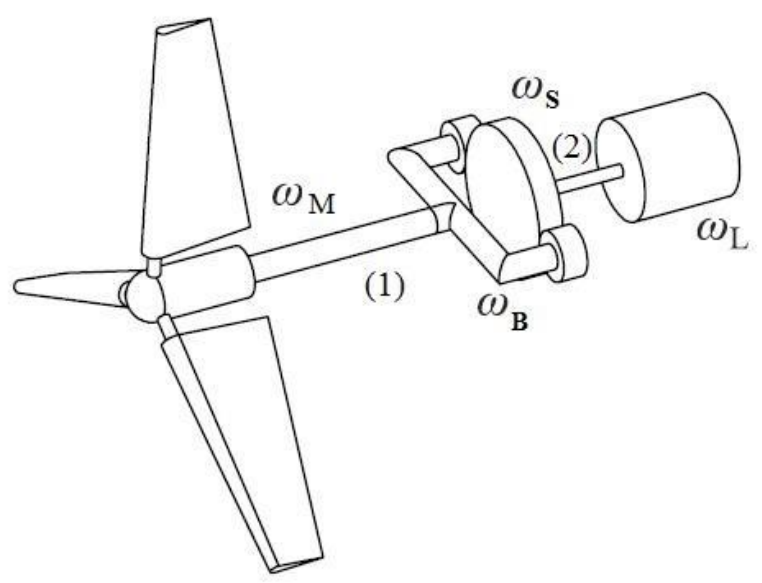

Fonte: acervo dos autores.

Para o momento de inércia de massa do gerador, inicialmente pode-se usar o modelo simples do momento de inércia de massa de um cilindro rotativo, cuja massa corresponde à massa das partes rotativas do gerador. Ressaltase que, em um modelo matemático dinâmico da turbina com um grau de liberdade, geralmente adota-se o eixo de baixa rotação como o eixo de referência e todas as inércias e momentos de inércias de elementos no eixo de alta rotação devem ser corrigidos. Portanto, o momento de inércia de massa equivalente do gerador pode ser dado por:

$$
J_{L \rightarrow T}=\frac{r^{2} J_{L}}{\eta}
$$

\section{CÓDIGO COMPUTACIONAL DESENVOLVIDO}

O modelo dinâmico mostrado na Equação (1) foi implementado em um código computacional denominado de DynTurb. O código é dividido em três partes, são elas: 1) cálculo do torque do rotor através da metodologia BEMT; 2) cálculo do torque dissipativo nos mancais, do torque de carga do gerador e dos momentos de inércia de massa do rotor, sistema de transmissão e gerador; 3) solução para o modelo dinâmico a partir do método Runge-Kutta de $4^{\mathrm{a}}$ ordem e análise dos resultados. Cada etapa é descrita a seguir. 
Para o cálculo do torque do rotor, desenvolveu-se um código com a metodologia BEMT. Nesse caso, é necessário se obter antes as características aerodinâmicas do perfil da pá. Assim, o usuário deve definir o tipo de perfil de pá a ser estudado. As principais características a serem definidas com a seleção do tipo de perfil são: 1) a geometria da pá, que engloba os dados para posição radial $r$, corda do perfil $c$ e ângulo de torção $\beta$; 2) coeficientes de sustentação $C_{L}$ e arrasto $C_{D}$, em função do ângulo de ataque $\alpha$; e 3) perfil de velocidade do fluido.

Outros parâmetros a serem considerados são: números de pás do rotor, massa específica do fluido $\left(\mathrm{kg} / \mathrm{m}^{3}\right)$, número máximo de iterações e tolerância para o ângulo de incidência $\emptyset$.

O fluxograma apresentado na Figura 3 ilustra o procedimento para o cálculo do torque do rotor. Primeiramente, arbitra-se valores para $a$ e $a$ '. No código, decidiu-se fixar esses valores, sendo $a=1 / 3$ e $a^{\prime}=0.02$, os quais podem ser alterados dentro do código. Com os parâmetros de entrada para o rotor já definidos pelo usuário, calcula-se os coeficientes normal $\left(C_{N}\right)$ e tangencial $\left(C_{T}\right)$, que representam, respectivamente, os esforços aerodinâmicos, empuxo e torque, no plano do rotor para cada posição radial. Somando os esforços exercidos em cada elemento de pá, obtém-se o valor total para o perfil. Nesse caso, o parâmetro de saída do programa será o torque do rotor, o primeiro termo do modelo dinâmico da turbina (Equação (1)). Durante o processo é aplicada a correção de Glauert (1935), que modifica o fator de indução axial, $a$, quando este atingir valores maiores que 0.4 , visto que o método falha nessas condições.

No código DynTurb, foi desenvolvida uma interface utilizando o GUIDE do Matlab. Ela possui uma faixa de opções com botões que acionam um painel para cada componente do modelo (Rotor, Trem de Potência, Análise). Dessa forma, o primeiro painel denominado ROTOR (Figura 4) é composto por componentes gráficos para entrada de dados do código com a metodologia BEMT e pelas saídas gráficas: o perfil da pá em 3D projetado pelo método BEMT (Figura 4), os coeficientes de sustentação e arrasto em função do ângulo de ataque e o perfil do fluxo de vento.

Figura 3 - Fluxograma para o código BEMT

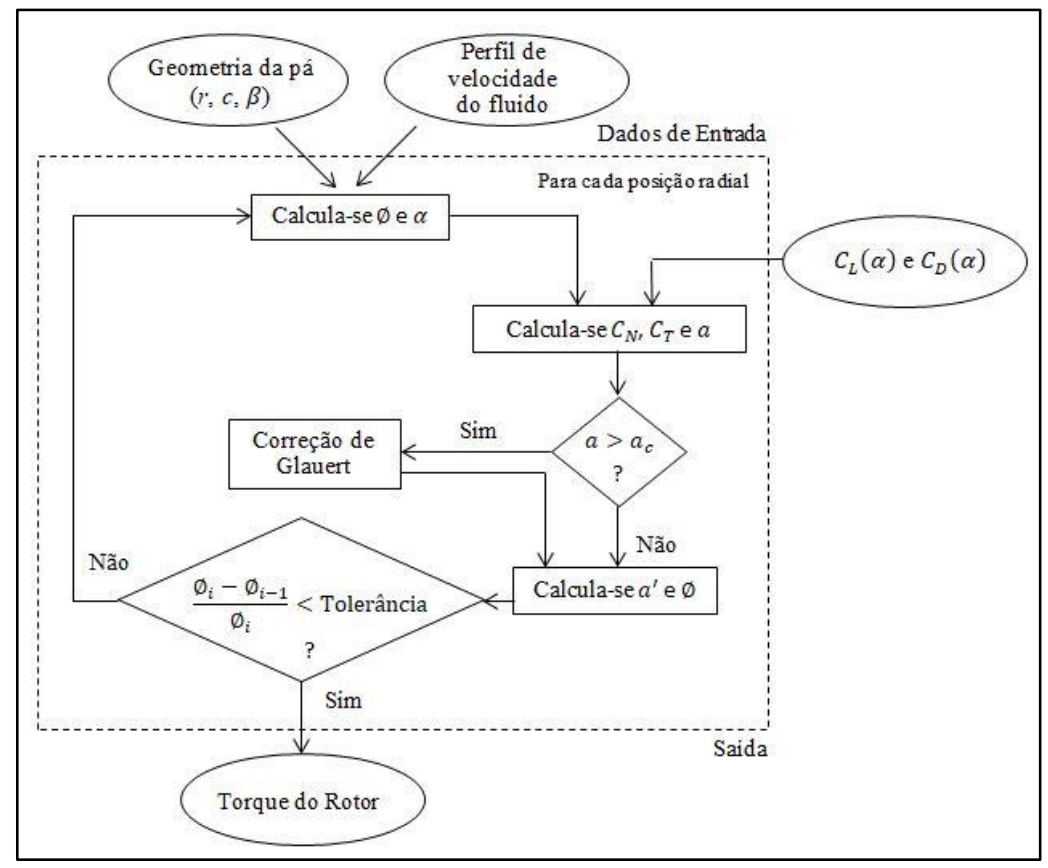

Fonte: acervo dos autores. 
Figura 4 - Painel para entrada de dados e visualização do perfil do rotor

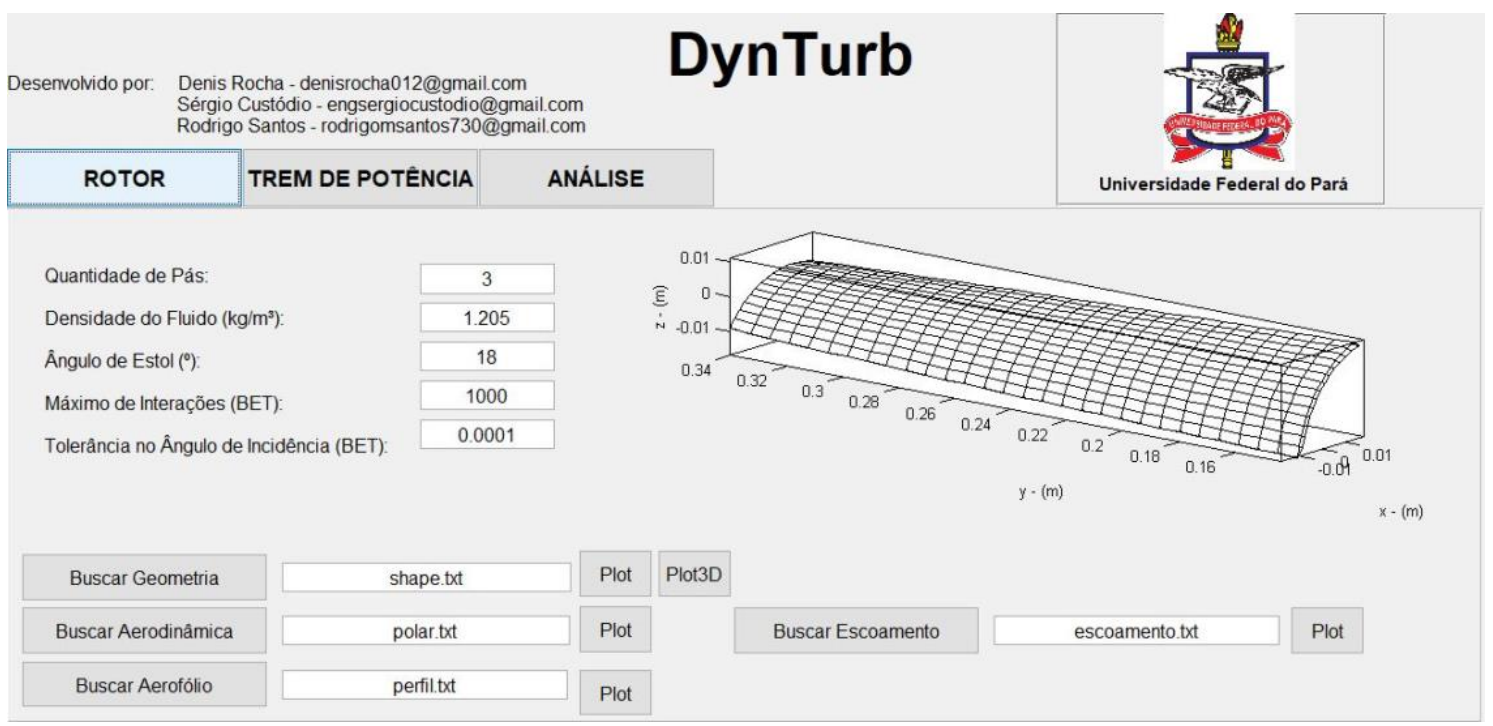

Fonte: acervo dos autores.

Figura 5 - Painel para o trem de potência

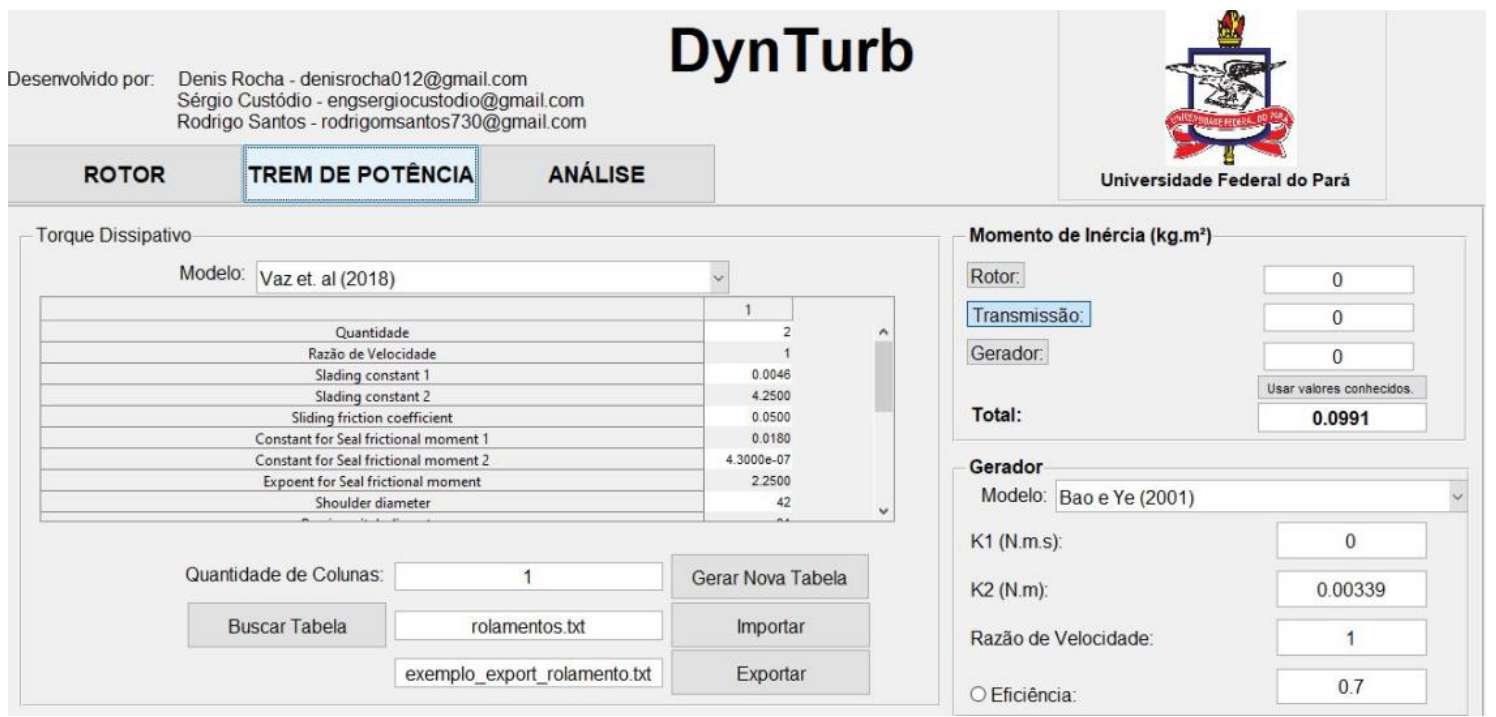

Fonte: acervo dos autores.

No painel para o trem de potência (Figura 5) são definidos o torque dissipativo, os momentos de inércia e o torque do gerador. Para o torque dissipativo pode ser selecionado o tipo de modelo para o seu cálculo, tais como modelo de Palmgren (1959), modelo SKF (2015) e modelo de Vaz et al. (2018). Por exemplo, selecionando-se o modelo de Vaz et al. (2018), surge uma tabela para inserção de dados (Figura 5). Nessa tabela são apresentadas as variáveis para esse modelo, com seus respectivos valores (que podem ser alterados). Além disso, nesse menu, pode ser selecionada a opção para adicionar outros modelos (equações) para o torque dissipativo conforme a necessidade do usuário.

Ainda nesse painel é permitido entrar com os valores dos momentos de inércia de massa para cada componente da turbina: rotor, sistema de transmissão e gerador. Para o sistema de transmissão foi desenvolvido um menu pop-up, a fim de selecionar os diferentes tipos de transmissão disponíveis ao usuário (Figura 6). Pode-se encontrar a transmissão por: engrenagens cilíndricas simples ou com dois estágios, engrenagens cônicas, engrenagem planetária e por correias. Ao selecionar o tipo de transmissão, uma nova janela será aberta ao 
usuário para a inserção dos dados, que são basicamente: o momento de inércia de massa de cada componente da transmissão e a razão de velocidades.

Figura 6 - Menu para seleção do tipo de transmissão

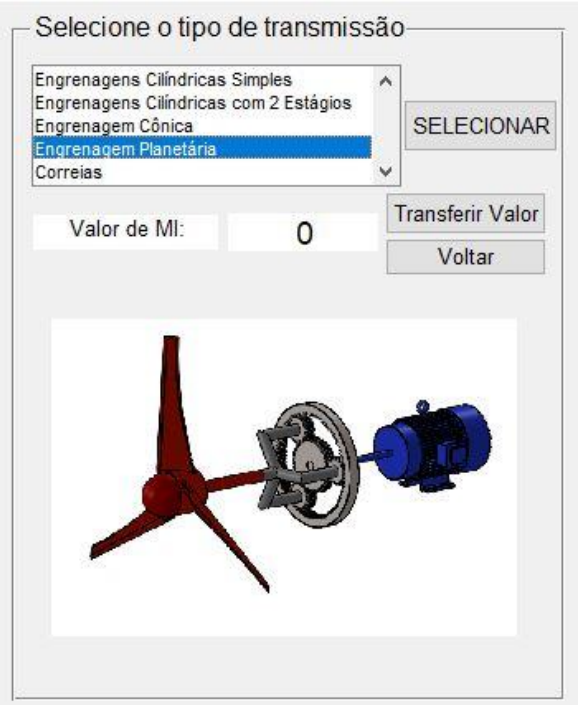

Para o torque do gerador também foi criado um menu pop-up para a escolha do seu modelo de cálculo, como exemplo o modelo proposto por Bao, Ye (2001), descrito anteriormente na Equação 14, além de se poder inserir outros modelos ou equações que regem o comportamento do tipo de gerador escolhido pelo usuário (Figura 5).

No painel para análise dos resultados (Figura 7) são encontrados alguns parâmetros (intervalo e passo de tempo da simulação) para solucionar a Equação (1) e também pode-se visualizar os resultados obtidos. A solução da Equação (1) é obtida pelo método de RungeKutta de $4^{\text {a }}$ ordem.

Fonte: acervo dos autores.

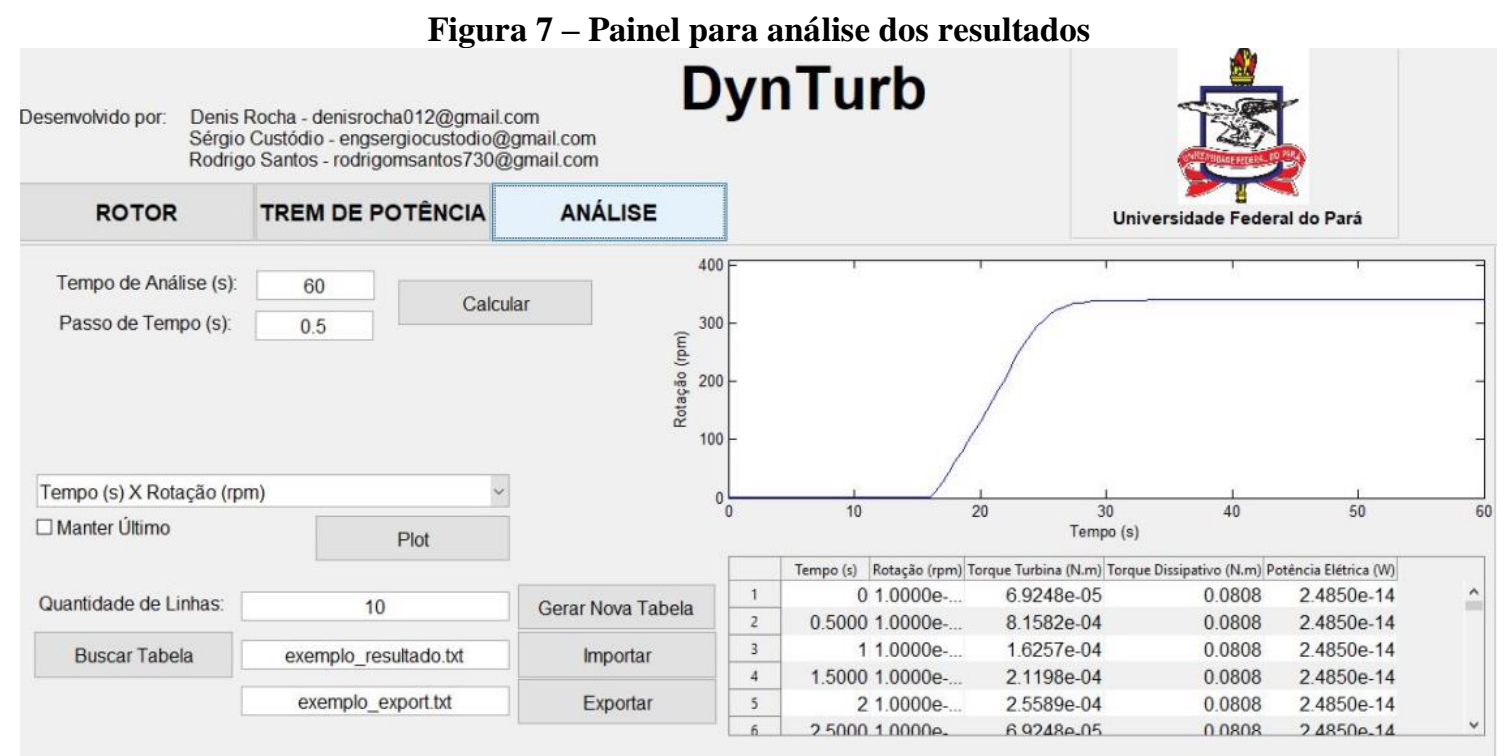

Fonte: acervo dos autores.

Os resultados que podem ser obtidos pelo programa são: rotação, torque do rotor, torque dissipativo e potência mecânica e elétrica em função do tempo, os quais podem ser selecionados através de um menu pop-up. Os resultados podem ser visualizados graficamente ou em uma tabela presente no painel, ou, ainda, serem exportados para um arquivo no formato txt, por exemplo.

\section{ESTUDO DE CASO}

Para verificar a funcionalidade do programa utilizou-se como referência a turbina descrita por Vaz et al. (2018).

\section{Descrição da Turbina}

A turbina consiste em um rotor com momento de inércia de massa, $J_{T}$, conectado a 
um eixo que é suportado por dois rolamentos rígidos de esferas (deep-groove ball bearings). No cálculo do torque dissipativo, foi considerada a metodologia descrita nas Equações (11) e (12), com seus parâmetros e respectivos valores para o Efeito de Stribeck. Por outro lado, os demais dados referentes ao modelo da SKF (2019), descritos na Equação (9) e utilizados na simulação, podem ser encontrados no próprio trabalho de $\mathrm{Vaz}$ et al. (2018). Esses dados foram organizados em arquivo .txt, para serem importados para a tabela no painel trem de potência da interface.

\section{Resultados}

Após todos os dados de entrada terem sido inseridos no programa DynTurb, foi obtida a curva de rotação do rotor em função do tempo. $\mathrm{O}$ resultado do programa forneceu exatamente a mesma curva apresentada por Vaz et al. (2018), conforme a Figura 8.

Figura 8 - Rotação em função do tempo.

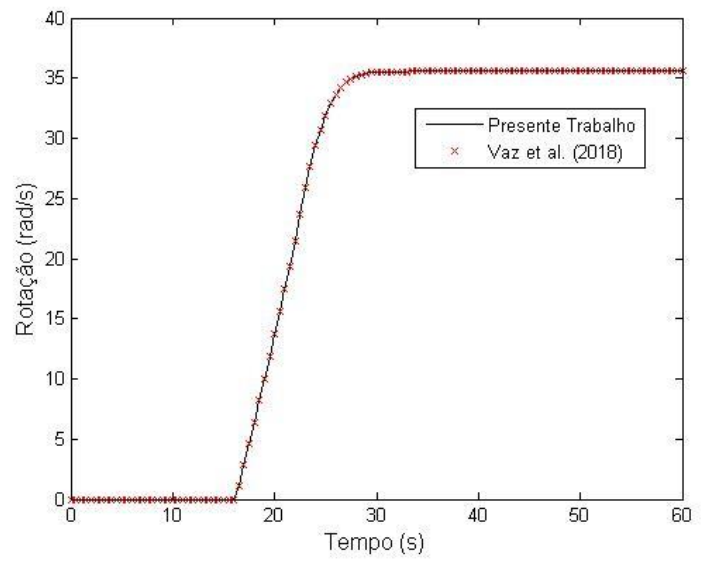

Fonte: acervo dos autores.

Muitos outros resultados podem ser obtidos devido à versatilidade do código computacional. Resultados como potência elétrica (Figura 9), torque do rotor e torque dissipativo em função do tempo também podem ser apresentados. Na Figura 10, por exemplo, percebe-se o intervalo de tempo em que o torque do rotor se torna maior que o torque dissipativo, em torno de 16 segundos. Quando o sistema atinge a marca de 31 segundos, o valor do torque do rotor torna-se bem próximo do valor do torque dissipativo, ou seja, o conjunto rotor-trem de potência entra em regime estacionário.

Um dos benefícios educacionais do programa é verificar facilmente o comportamento dinâmico da turbina ao se variar alguns dos seus parâmetros. Por exemplo, pode-se visualizar que ao aumentar o torque resistente do sistema (torque dissipativo nos rolamentos + torque de carga no gerador), isto vai provocar uma redução na rotação do rotor, como visto na Figura 11. Um outro exemplo seria variar o momento de inércia do sistema e verificar o que irá resultar no movimento da turbina. A Figura 12 mostra esse efeito, ou seja, com o aumento do momento de inércia de massa total, o rotor demorará mais tempo para atingir o regime permanente, ou seja, o regime transiente será maior.

Figura 9 - Potência elétrica em função do tempo

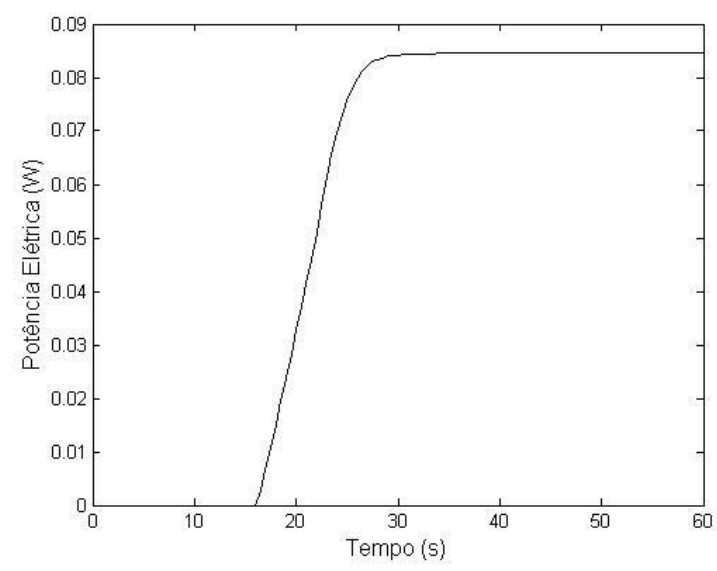

Fonte: acervo dos autores.

Figura 10 - Torque em função do tempo

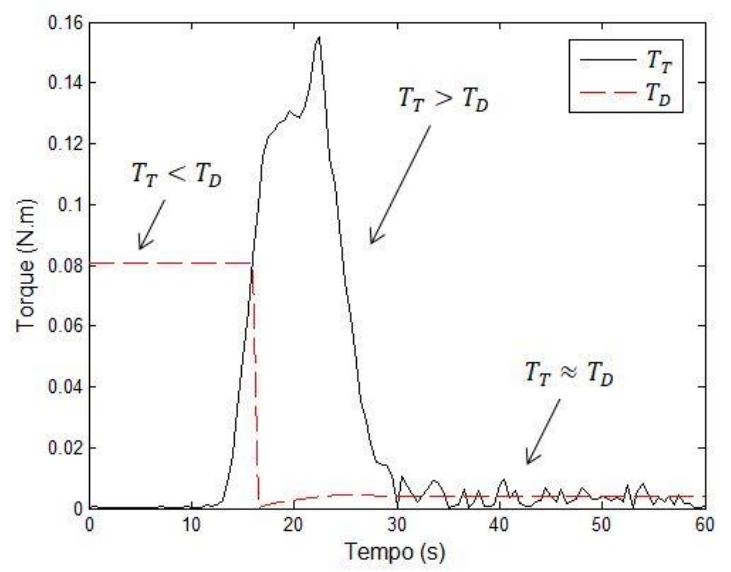

Fonte: acervo dos autores. 
Figura 11 - Rotação x tempo, variando o torque resistente

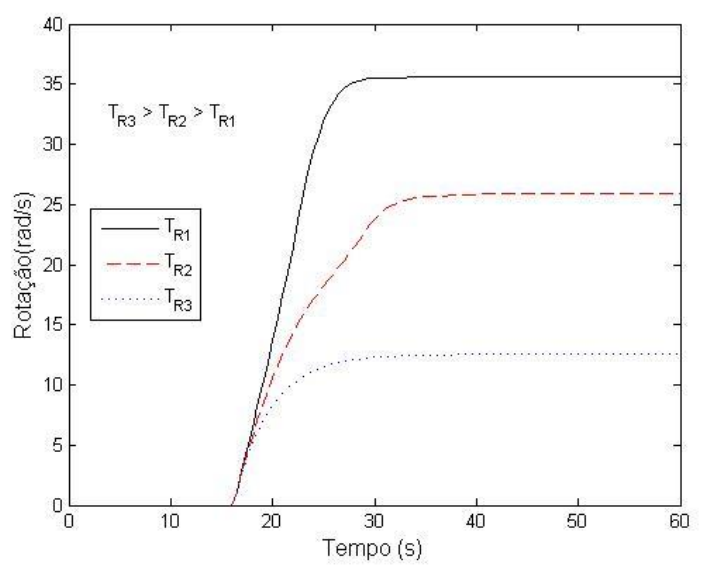

Fonte: acervo dos autores.

Figura 12 - Rotação x tempo, variando o momento de inércia de massa total

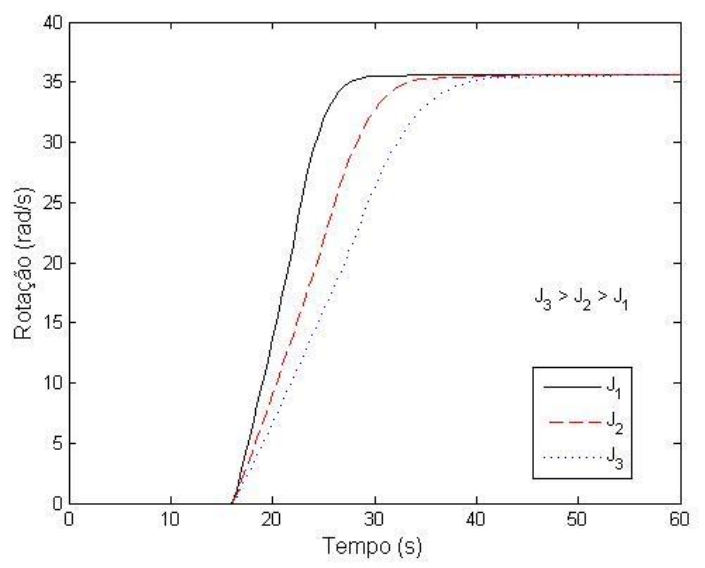

Fonte: acervo dos autores.

\section{CONCLUSÃO}

Este artigo apresentou o desenvolvimento do código computacional DynTur, escrito em ambiente Matlab, para fins educacionais e de pesquisa no tema de dinâmica de turbinas cinéticas (eólicas e hidrocinéticas) de eixo horizontal. O programa leva em conta o acoplamento rotor com seu trem de potência, em que os efeitos de inércia, resistência por atrito e carga do gerador são inclusos.

Esse programa computacional com suas interfaces gráficas é de fácil utilização, o que proporciona uma fácil modelagem de turbinas cinéticas e também se torna uma ferramenta eficaz para $\mathrm{o}$ processo de ensino $\mathrm{e}$ aprendizagem do tema. Com a possibilidade de inserção de novos modelos matemáticos de torque dissipativos, torque de carga do gerador e de momentos de inércia, o programa também auxilia na investigação de novos modelos matemáticos.

\section{AGRADECIMENTOS}

À Pró-Reitoria de Pesquisa e PósGraduação (PROPESP/UFPA) pela coordenação de atividades e programas pertinentes à sua área de atuação e à Coordenação de Aperfeiçoamento de Pessoal de Nível Superior Brasil (CAPES) pelo apoio financeiro.

\section{REFERÊNCIAS}

BAO, N.; YE, Z. Active Pitch Control in Larger Scale Fixed Speed Horizontal Axis Wind Turbine Systems Part I: Linear Controller Design. Wind Engineering, v. 25, n. 6, p. 339-351, 2001.

BLASQUES, L. C. et al. Caracterização da potencialidade eólica e análise comparativa entre diferentes aerogeradores para localidades costeiras das regiões norte e nordeste do Brasil. Anais... III Congresso Brasileiro de Energia Solar, Belém - Pará, Brasil, 2010.

BRÄNDLEIN, J. et al. Ball and roller bearings: theory, design and application. Wiley, 1999.

DEHOUCK, Vincent et al. Application of the Blade Element Momentum Theory to Design Horizontal Axis Wind Turbine Blades. Journal of Solar Energy Engineering, v. 140, n. 1, 2018.

FIGUEIREDO, S. W. O. et al. Protótipo hidrocinético para geração de energia elétrica aplicado à educação em engenharia. Revista de Ensino de Engenharia, v. 32, n. 1, p. 19-30, 2013. 
GLAUERT, H. Airplane propellers. In: Aerodynamic theory. Springer, Berlin, Heidelberg, 1935.

HANSEN, M. O. L. Aerodynamics of wind turbines. Earthscan: London, 2008.

IEMA. IEMA estuda alternativas para 500 mil brasileiros sem acesso à eletricidade na Amazônia. 2009. Disponível em: $<$ http://www.energiaeambiente.org.br/iem a-estuda-alternativas-para-500-milbrasileiros-sem-acesso-a-eletricidade-naamazonia>. Acesso em: 30 mai. 2019.

KUMAR, D.; SARKAR, S. Areview on the technology, performance, design optimization, reliability, techno-economics and environmental impacts of hydrokinetic energy conversion systems. Renewable and Sustainable Energy Reviews, v. 58, p. 796-813, 2016.

LAWS, N. D.; EPPS, B. P. Hydrokinetic energy conversion: Technology, research, and outlook. Renewable and Sustainable Energy Reviews, v. 57, p. 1245-1259, 2016.

LEWIS, F. M.; AUSLAENDER, Jm. Virtual inertia of propellers. Journal of Ship Research, v. 3, n. 4, p. 37-46, 1960.

LIMA, W. M. et al. Uma ferramenta computacional para suporte nos processos de ensino e aprendizagem de equações diferenciais parciais. Revista de Ensino de Engenharia, v. 35, n. 1, 2016.

LOPES, J. J. A. et al. An Approach for the Dynamic Behavior of Hydrokinetic Turbines. Energy Procedia, v. 75, p. 271276, 2015.

MANIACI, D. C.; LI, Y. Investigating the influence of the added mass effect to marine hydrokinetic horizontal-axis turbines using a General Dynamic Wake wind turbine code. Marine Technology
Society Journal, v. 46, n. 4, p. 71-78, 2012.

MARIANI, V. C.; MARTIM, E. Ferramentas computacionais na sala de aula: minimizando o descompasso entre conhecimento acadêmico e realidade. Revista de Ensino de Engenharia, v. 26, n. 1, 2008.

MESQUITA, A. L. A. et al. A methodology for the transient behavior of horizontal axis hydrokinetic turbines. Energy conversion and management, v. 87 , p. 1261-1268, 2014.

PALMGREN, A. Ball and roller bearing engineering. Philadelphia: SKF Industries Inc., 1959.

REDDY, G. P. K; BHAGYAMMA, S. S. D. S. Fixed-Speed and Variable Speed (PMSG) Induction Generators Based Wind Farms with Statcom Control under Asymmetrical Grid Faults, 2014.

ROSALES, P. et al. Comparative Assessment of a Horizontal Small Wind Turbine with Ball and Magnetic Bearings on the Starting. Chemical Engineering, v. 34, 2013.

SILVA, P. A. S. F. et al. Analysis of cavitation for the optimized design of hydrokinetic turbines using BEM. Applied energy, v. 185, p. 1281-1291, 2017.

SKF. Catálogo Geral 10000_2. 2015. Disponível

em:<https://www.skf.com/binaries/pub45/ Images/0901d19680416a2b-10000_2-PT-

BR---Rolling-bearings_tcm_45-

121486.pdf>. Acesso em: 30 mai. 2019.

VAN ELS, R. H; JUNIOR, A. C. P. B. The Brazilian experience with hydrokinetic turbines. Energy Procedia, v. 75, p. 259264, 2015. 
VÁSQUEZ, F. A. M.; DE OLIVEIRA, T. F.; JUNIOR, A. C. P. B. On the electromechanical behavior of hydrokinetic turbines. Energy Conversion and Management, v. 115, p. 60-70, 2016.

VAZ, J. R.P.; WOOD, D. H. Effect of the diffuser efficiency on wind turbine performance. Renewable energy, v. 126, p. $969-977,2018$.
VAZ, J. R. P et al. Drivetrain resistance and starting performance of a small wind turbine. Renewable energy, v. 117, p. 509$519,2018$.

WILSON, W. K. Practical solution of torsional vibration problems: with examples from marine, electrical, aeronautical, and automobile engineering practice. Chapman \& Hall: UK, 1956.

\section{DADOS BIOGRÁFICOS DOS AUTORES}
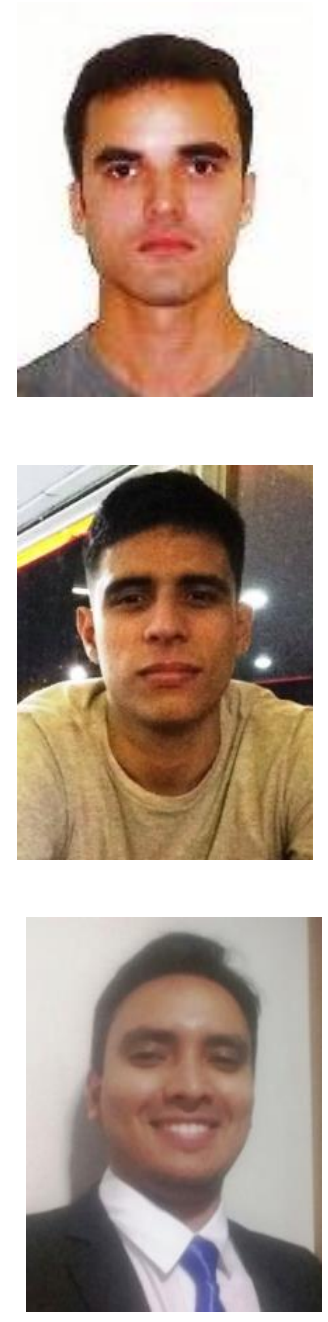

Denis Melo Rocha possui graduação em Engenharia Mecânica pela Universidade Federal do Pará (2011), com experiência profissional na área de Energia e Transporte de Minérios. Atualmente é aluno bolsista de Mestrado em Engenharia Mecânica no PPGEM/UFPA.

Rodrigo Marques dos Santos é graduando em Engenharia Mecânica pela Universidade Federal do Pará. Atualmente é integrante do Grupo de Vibrações e Acústica (UFPA) e bolsista no Programa de Educação Tutorial (PET).

Sérgio de Souza Custódio Filho possui graduação em Engenharia Mecânica pela UFPA (2016), com experiência internacional como bolsista do Programa BRAFITEC, em Paris - França, participando do programa de Especialização em Engenharia de Segurança do Trabalho pela UFPA (2019). Atualmente é aluno de Mestrado em Engenharia Mecânica no PPGEM/UFPA e Professor Substituto na Faculdade de Engenharia Mecânica da UFPA. 


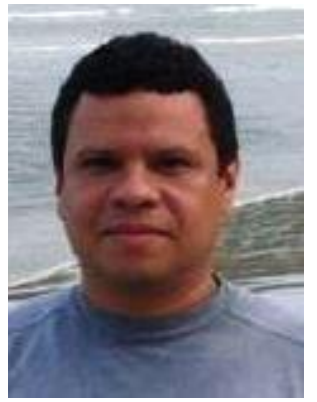

Alexandre Luiz Amarante Mesquita tem Doutorado em Engenharia Mecânica pela Universidade Estadual de Campinas (2004). Atualmente é Professor Associado IV do Curso de Engenharia Mecânica na Universidade Federal do Pará. Possui experiência na área de Dinâmica Aplicada, atuando principalmente nos seguintes temas: Vibrações, Análise Modal, Dinâmica de Rotores, Processamento de Sinais, Acústica, Projeto de Máquinas, Transporte de Minérios e Método dos Elementos Discretos. 